Article

\title{
Non-Gaussian Distributions to Random Walk in the Context of Memory Kernels
}

\author{
Maike A. F. dos Santos \\ Instituto de Física, Universidade Federal do Rio Grande do Sul, Caixa Postal 15051, CEP 91501-970 Porto Alegre, \\ RS, Brazil; santos.maike@ufrgs.br
}

Received: 13 July 2018; Accepted: 27 July 2018; Published: 29 July 2018

\begin{abstract}
The investigation of diffusive process in nature presents a complexity associated with memory effects. Thereby, it is necessary new mathematical models to involve memory concept in diffusion. In the following, I approach the continuous time random walks in the context of generalised diffusion equations. To do this, I investigate the diffusion equation with exponential and Mittag-Leffler memory-kernels in the context of Caputo-Fabrizio and Atangana-Baleanu fractional operators on Caputo sense. Thus, exact expressions for the probability distributions are obtained, in that non-Gaussian distributions emerge. I connect the distribution obtained with a rich class of diffusive behaviour. Moreover, I propose a generalised model to describe the random walk process with resetting on memory kernel context.
\end{abstract}

Keywords: fractional diffusion equation; memory kernels; random walk; diffusion models; solution techniques; anomalous diffusion

PACS: 05.40.-a; 05.10.Gg; 05.40.Fb; 46.65.+g

\section{Introduction}

The random walk formalism is a fundamental tool to approach fluctuations associated to atoms and molecules. In this sense, the random walk is essential on description of stochastic process in statistical physics. In the context of the random walk, the Brownian motion investigated by Einstein can be associated with stochastic process caused by molecules of fluid [1]; this hypothesis was also investigated by Smoluchowski [2] and Langevin [3], and confirmed by Jean Perin's experiments [4,5]. In this framework, a class of random walkers that is Markovian and ergodic may be connected with Gaussian distribution and usual diffusion, i.e., $\left\langle(\Delta x)^{2}\right\rangle=2 d \mathcal{D} t$, in which $d$ spacial dimension and $\mathcal{D}$ is diffusion coefficient. In other words, the mean square displacement (MSD) assumes a linear behaviour in time; the anomalous diffusion defines the process in which occur the break of linear temporal behaviour to MSD. Recently, there are several investigations that reported the anomalous diffusion process in complex systems, e.g., we have anomalous diffusion in biological systems [6-8], and in fractal or disordered structures [9-12]. The anomalous diffusion implies a mean square displacement characterized by $\left\langle(x-\langle x\rangle)^{\rangle} \propto t^{\alpha}\right.$, in which it can be classified as super-diffusion to $\alpha>1$ and sub-diffusion to $\alpha<1$. In this scenario, a rich class of non-Gaussian distributions emerges. Thus, there are two types of ordinary formalisms that imply non-Gaussian distributions associated to anomalous spreading, the non-linear equations and fractional equations. The non-linear diffusion recovers the usual diffusion as a particular case. In this framework, the non-linear diffusion equation has been investigated in quantum random walk [13], random walks [14], and Fokker-Planck equation [15-17].

The fractional diffusion can be justified by fractional calculus which involves the non-integer order to derivative and integral [18]. These quantities are essentially defined by convolutions integrals with kernel of type power law functions that define the well-know Caputo, Riemann-Liouville 
and Riesz-Feller fractional operators, which are focus of recent mathematical applications [19-26]. Nowadays, a new family of kernels has been used to describe generalised diffusive models [27-34]. In this context, it is possible to define the generalised diffusion equation

$$
\int_{0}^{t} d t^{\prime} \mathcal{K}\left(t-t^{\prime}\right) \frac{\partial \rho}{\partial t^{\prime}}=\mathcal{D} \frac{\partial^{2} \rho}{\partial x^{2}}, \quad-\infty<x<+\infty,
$$

in which $\mathcal{D}$ is a generalised diffusion coefficient. Noting that $\mathcal{K}(t)=\delta(t)$ recovers the usual diffusion equation. Equation (1) describes a free diffusion (diffusion with memory kernel with conditions $\rho( \pm \infty, t)=0$ and $\rho(x, 0)=\varphi(x)$. The choice of kernel $\mathcal{K}(t)$ defines the structure of temporal derivative. On the other hand, a recent theme on literature is searching the best way to include memory kernels in the diffusion equation [35-37]. The memory kernel as put on Equation (1) was considered on Fokker-Planck equation [38], the memory kernel in Laplacian term was considered in Ref. [36]. However, establishing the scenario which contains the exact solution to Equation (1) of some kernels is necessary.

The standard fractional form consider the singular power law kernel, as

$$
\mathcal{K}(t)=\frac{t^{-\alpha}}{\Gamma[1-\alpha]}, \quad \alpha \in(0,1),
$$

in which Equation (1) incorporates the well-known Caputo fractional operator to temporal variable for $0<\alpha<1$. To consider these situations, two different forms of non-singular kernels $\mathcal{K}(t)$ are considered. The first one decays as a exponential form

$$
\mathcal{K}(t)=\frac{f}{\tau} \exp \left[-\frac{t}{\tau}\right], \quad \tau \in[0,+\infty]
$$

in which $f$ denotes a arbitrary constant. Assuming the particular choices $f=\frac{f_{\alpha}}{\alpha}$ and $\tau=\frac{1-\alpha}{\alpha}$, we obtain a special kernel which defines the fractional Caputo-Fabrizio derivative [32] in Equation (1) that, for simplicity, I denominate Caputo-Fabrizio diffusion.

The second one is

$$
\mathcal{K}(t)=\frac{b}{\tau} E_{\alpha, 1}\left[-\frac{t^{\alpha}}{\tau}\right], \quad \alpha \in(0,1)
$$

$\tau \in[0,+\infty]$, in which $E_{\alpha, \gamma}(\cdots)$ denotes the Mittag-Leffler function for two parameters, i.e.,

$$
E_{\alpha, \gamma}(z)=\sum_{l=0}^{\infty} \frac{z^{l}}{\Gamma[l \alpha+\gamma]}
$$

in which $E_{\alpha, 1}=E_{\alpha}$. To particular choice $b=\frac{b_{\alpha}}{\alpha}$ and $\tau=\frac{1-\alpha}{\alpha}$, Equation (4) assumes the kernel which defines the fractional Atangana-Baleanu derivative [31] in Equation (1). For simplicity, I denominate Atangana-Baleanu diffusion.

Equations (3) and (4) are non-singular forms (i.e., $\lim _{x \rightarrow 0} \mathcal{K}(x) \neq+\infty$ ). Here, one question may be considered: how can the non-singular kernel in diffusion equation (Equation (1)) be linked with continuous time random walk (CTRW)? To answer this question, it is necessary to introduce stochastic quantities to random walkers. The CTRW is a formalism proposed by Montroll and Weiss in 1965 to describe a walk using joint distribution of jump length and waiting time $\Psi(x, t)$ [39-41], in which $\psi(t)=\int_{-\infty}^{+\infty} d x \Psi(x, t)$ is the waiting time distribution of a walker between two successive jumps and $\lambda(x)=\int_{0}^{+\infty} d t \Psi(x, t)$ is the distribution of jump length. This formalism implies a class of fractional diffusion equation. The Montroll-Weiss equation has several consequences in temporal memory and non-local behaviour in transport system in disordered medium and complex system [42-44]. The appropriate choice for waiting time distribution describes systems such as integration-differential 
equation with chemical interaction [24,45,46], anomalous diffusion in expanding media [30] and diffusion of single metal atoms on a graphene oxide support [47].

The main objective of this work is to obtain the exact solutions to diffusion equation in generalised context for memory kernels and to present an applicability on the model of diffusion with stochastic resetting. Section 2 presentd the preliminary topics, including Montroll-Weiss equation on random walk movement with memory effects. Section 2.2 approaches how this memory kernel are connected with different versions of the diffusion equation. Consequently, in Section 3, I divide the results into four subsections: firstly, I consider the exponential kernel in Equation (1) and I obtain the exact solution by mean of Laplace-Fourier transforms. Secondly, I show the exact solution to diffusion equation under the presence of Mittag-Leffler memory kernel. The results imply non-Gaussian shapes due to memory effects. Thirdly, the anomalous relaxation processes associated with generalised random walks are discussed. Finally, I exemplify the efficiency of the methods proposed here, finding generalised solutions to the problem of diffusion with stochastic resetting. Section 4 presents the conclusions.

\section{Preliminaries}

We review some notions and concepts used throughout the paper.

\subsection{Montroll-Weiss Formalism}

The Montroll-Weiss equation establishes the distribution of probability in Laplace-Fourier space of random walks, as follows

$$
\rho(k, s)=\frac{1-\psi(s)}{s} \frac{\varphi(k)}{1-\Psi(k, s)},
$$

as defined in Ref. [43]. In the case the joint distribution of jump length and waiting time is separable, i.e., $\Psi(k, s)=\psi(s) \lambda(k)$, the choice $\lambda(k) \sim 1-k^{2} \mathcal{D} \tau$, in that $\tau$ is a characteristic waiting time of the underlying continuous time random walk. It is important observe that the jump probability distribution is characterized by a Gaussian asymptotic behaviour, i.e., $\lambda(x) \sim e^{-\frac{x^{2}}{(2 \mathcal{D} \tau)^{2}}}$, and it has a finite characteristic jump length. If $\psi(t)=\frac{e^{-\frac{t}{\tau}}}{\tau}$ (Poissonian distribution), we obtain the free diffusion (Brownian motion). For $\psi(t) \sim A_{\alpha}(\tau / t)^{1+\alpha}$, it is possible to write the diffusion equation with fractional Riemann-Liouville operator [48]. To include a generalised situation to waiting time, consider the next waiting time distribution in Laplace space

$$
\psi(s)=\frac{1}{\tau s \mathcal{K}_{L}(s)+1},
$$

as proposed in Ref. [38], in which $\mathcal{L}\{\mathcal{K}(t)\}=\int_{0}^{\infty} e^{-s t} \mathcal{K}(t) d t=\mathcal{K}_{L}(s)$ denote the Laplace transform of memory kernel and $\tau$ is characteristic time. For $\mathcal{K}(t)=\delta(t)$, we recover the Brownian motion. The kernel implies a class of generalised diffusion random walks, therefore Equation (7) in Montroll-Weiss equation implies

$$
\begin{aligned}
\rho(k, s) & =\frac{\tau s \mathcal{K}_{L}(s)}{s} \frac{\varphi(k)}{\tau s \mathcal{K}_{L}(s)+1-\left(1-k^{2} \mathcal{D} \tau\right)} \\
& =\frac{\mathcal{K}_{L}(s) \varphi(k)}{s \mathcal{K}_{L}(s)+k^{2} \mathcal{D}} .
\end{aligned}
$$

It is now necessary to emphasize two important points that are subtle and fundamental to the continuity of work. First, we approach the waiting time on Sandev sense [38]; there are alternative forms in the literature, as discussed in the Section 1. In fact, the choice of different forms to waiting time distribution change how the memory kernel appears on diffusion equation [49]. Second, it is important to find exact solutions to the generalised diffusion equation, which has attracted the attention of many researchers. 
Equation (8) is a density distribution of walkers in Fourier-Laplace space, and includes the arbitrary kernel present waiting time distribution in CTRW formalism. Considering the kernel memory of Caputo operator in Laplace space, i.e., $\mathcal{K}_{L}(s)=s^{\alpha-1}$, waiting time distribution implies a particular form

$$
\psi(t)=\frac{t^{\alpha-1}}{\tau} E_{\alpha, \alpha}\left[-\frac{t^{\alpha}}{\tau}\right] .
$$

in which $\mathcal{L}\left\{t^{\gamma-1} E_{\alpha, \gamma}\left(-\lambda t^{\alpha}\right)\right\}=\frac{s^{\alpha-\gamma}}{\lambda+s^{\alpha}}$.

For standard fractional Caputo operator, i.e., $\mathcal{K}_{L}(s)=s^{\alpha-1}$, the Fourier-Laplace inversion implies the following solution

$$
\rho(x, t)=\int_{-\infty}^{+\infty} d x^{\prime} \frac{\varphi\left(x^{\prime}\right)}{\sqrt{4 t^{\alpha} \mathcal{D}_{\alpha}}} \mathrm{H}_{1,1}^{1,0}\left[\left.\frac{\left|x-x^{\prime}\right|}{\mathcal{D}_{\alpha}^{\frac{1}{2}} t^{\frac{\alpha}{2}}}\right|_{(0,1)} ^{\left(1-\frac{\alpha}{2}, \frac{\alpha}{2}\right)}\right],
$$

The $H(\cdots)$ is Fox function. The Fox function consists in a type of Merlin integration [50]. This function is a characteristic type of solutions to fractional diffusion equations. Equation (10) has a non-Gaussian shape to $\alpha \neq 1$, which was investigated in the context of fractional dynamics by Metzler and Klafter in [43].

The Mittag-Leffler kernel in waiting time distribution reveals an intermediate behaviour between power law and stretched exponential functions. This case was discussed in References [36,49]. Equation (8) reports an important generalisation of the diffusion equation in fractional derivative context, which is the theme of the next subsection.

\subsection{Generalised Diffusion Equation}

The exponential and Mittag-Leffler kernels in Montroll-Weiss equation can imply other forms of the diffusion equation. These forms are relevant to conduction phenomena and transport process. The Equation (8) can be rewritten as follow

$$
s \mathcal{K}_{L}(s) \rho(k . s)-\mathcal{K}_{L}(s) \varphi(k)=-\mathcal{D}_{\alpha} k^{2} \rho(k, s) .
$$

It is important to notice that the inversion in Laplace-Fourier transforms imply the general diffusion in Equation (1). On the other hand, for the exponential kernel (Equation (3)) in Equation (11), we can rewrite an alternative expression

$$
\frac{\partial \rho}{\partial t}=\mathcal{D} \frac{\partial^{2} \rho}{\partial x^{2}}+\mathcal{D}_{\tau} \frac{\partial^{3} \rho}{\partial t \partial x^{2}}
$$

in which $\mathcal{D}_{\tau}=\tau \mathcal{D}$. This equation was investigated for the seepage of a homogeneous fluid in fissured rocks, by Barenblatt [51]. From the Mittag-Leffler kernel in (Equation (4)) in Equation (11), we obtain

$$
{ }_{0}^{C} \mathrm{D}_{t}^{\alpha} \rho=\mathcal{D} \frac{\partial^{2} \rho}{\partial x^{2}}+{ }_{0}^{R L} \mathrm{D}_{t}^{\alpha} \mathcal{D}_{\tau} \frac{\partial^{2} \rho}{\partial x^{2}} .
$$

This equation is a natural extension of Equation (12) for the fractional case. The fractional operators are defined by

$$
{ }_{0}^{C} \mathrm{D}_{t}^{\alpha} g(t)=\frac{1}{\Gamma[1-\alpha]} \int_{0}^{t}\left(t-t^{\prime}\right)^{-\alpha} \frac{d g}{d t^{\prime \prime}}
$$

which is the Caputo fractional operator for $0<\alpha<1$ [18], and

$$
{ }_{0}^{R L} \mathrm{D}_{t}^{\alpha} g(t)=\frac{1}{\Gamma[1-\alpha]} \frac{d}{d t} \int_{0}^{t}\left(t-t^{\prime}\right)^{-\alpha} g\left(t^{\prime}\right)
$$


which is the Riemann-Liouville operator for $0<\alpha<1$ [18]. However, the memory effects in random walk context imply a class of generalised diffusion equation written in terms of Riemann-Liouville and Caputo operators. Equations (12) and (13) are formed by fractional derivative with singular kernels but are connected which non-singular representation, i.e., Equation (1). This analysis suggests that the Caputo and Riemann-Liouville fractional operator can represent a more general class of diffusive phenomena.

\section{Main Results}

In this section, the objective is to investigate formal solutions for the fractional diffusion in Equation (1) when the two different memory kernel are considered. The intention is to show that the memory functions in the diffusion equation imply a rich class of non-Gaussian forms to spatial distribution. These solutions are obtained by performing the inverse of Fourier and Laplace transforms of Montroll-Weiss equation, expressed by Equation (8). Performing the Fourier transform in the spatial variable, we obtain the general solution in terms of convolution form:

$$
\rho(x, s)=\int_{-\infty}^{+\infty} d x^{\prime} \varphi\left(x^{\prime}\right) \mathcal{G}\left(x-x^{\prime}, s\right),
$$

in which the $\mathcal{G}$-function (Green function) is

$$
\mathcal{G}(x, s)=\sqrt{\frac{\mathcal{K}(s)}{4 s \mathcal{D}_{\alpha}}} \exp \left[-\sqrt{\left.\frac{\mathcal{K}(s) s}{\mathcal{D}_{\alpha}}|x|\right] .}\right.
$$

Now, I separate into subsections to approach the solution in more details.

However, for non-singular operators acting in diffusion equation, the inversion of Laplace transform is not an easy task. Therefore, for the next Laplace-inverse transforms, the integrations are performed in $s$ complex plane to find the non-Gaussian distributions. In addition, I establish the diffusive analysis approaching the anomalous diffusion behaviour that appears. Moreover, I generalise the diffusive green function, i.e., Equation (17), to the context of diffusion with stochastic resetting.

\subsection{Exponential Memory-Kernel and Non-Gaussian Solutions}

Assuming the exponential kernel. i.e., Equation (3), performing the Laplace transform, one obtains

$$
\mathcal{K}(s)=\frac{f}{\tau s+1}, \quad \tau \in[0,+\infty]
$$

In context of fractional operator of Caputo-Fabrizio, we have

$$
f=\frac{f_{\alpha}}{\alpha} \quad \text { and } \quad \tau=\frac{1-\alpha}{\alpha},
$$

in which $f_{\alpha}$ denotes a normalization constant and $\alpha \in(0,1)$. The Green function can be rewritten as

$$
\mathcal{G}(x, s)=-\frac{1}{2} \frac{\partial Y}{\partial|x|}
$$

in which

$$
Y(x, s)=\frac{1}{s} \exp \left[-\frac{|x|}{\sqrt{\tau \mathcal{D}}} \sqrt{\frac{s}{s+\frac{1}{\tau}}}\right],
$$

taking the branch cuts associated with $\sqrt{s}$ and $\sqrt{s+\frac{1}{\tau}}$ along the negative real axis of s-plane (for more details, see [52]), the inversion is 


$$
\mathrm{Y}(x, t)=1-\frac{2}{\pi} \int_{0}^{\infty} d \eta \exp \left[-\frac{1}{\tau} \frac{t \eta^{2}}{\eta^{2}+1}\right] \frac{\sin \left[\frac{|x|}{\sqrt{\tau \mathcal{D} f^{-1}}} \eta\right]}{\eta\left(1+\eta^{2}\right)} .
$$

However, using the definition in Equation (20), the $\mathcal{G}$-function corresponding to the kernel in Equation (3) is

$$
\mathcal{G}_{1}(x, t)=\frac{1}{\pi \sqrt{\tau \mathcal{D} f^{-1}}} \int_{0}^{\infty} d \eta \exp \left[-\frac{1}{\tau} \frac{t \eta^{2}}{\eta^{2}+1}\right] \frac{\cos \left[\eta \frac{|x|}{\sqrt{\tau \mathcal{D} f^{-1}}}\right]}{1+\eta^{2}} .
$$

Equations (16) and (23) correspond to solution of diffusion equation with exponential kernel. In the case all walks are localized in a single point, i.e., $\rho(x, 0)=\delta(x)$. It implies $\rho(x, t)$ that is governed by Equation (23); this distribution in space is illustrated in Figure 1 to a set of fractional index. Figure 2 exemplifies the temporal evolution of non-Gaussian distribution on time, to exponential memory. Moreover, the special situation in Equations (19) and (23) assumes the exact green function to solution of Caputo-Fabrizio diffusion.



Figure 1. This curves illustrate the changes of distribution $\rho(x, t)$ caused by the exponential kernel (Equation (3) in Equation (1)), and the usual random walk (Brownian motion) case $\mathcal{K}(t)=\delta(t)$ (or $\tau=0$ in Equation (3)), considering $\varphi(x)=\delta(x), \mathcal{D} f^{-1}=1, t=1$, and a family of characteristic times $\left\{10^{-2}, 10^{-1}, 0.5,1,2,4\right\}$ (in context Caputo-Fabrizio operator $\tau=(1-\alpha) \alpha^{-1}$ and $\mathcal{D} f^{-1}=1$ ). 


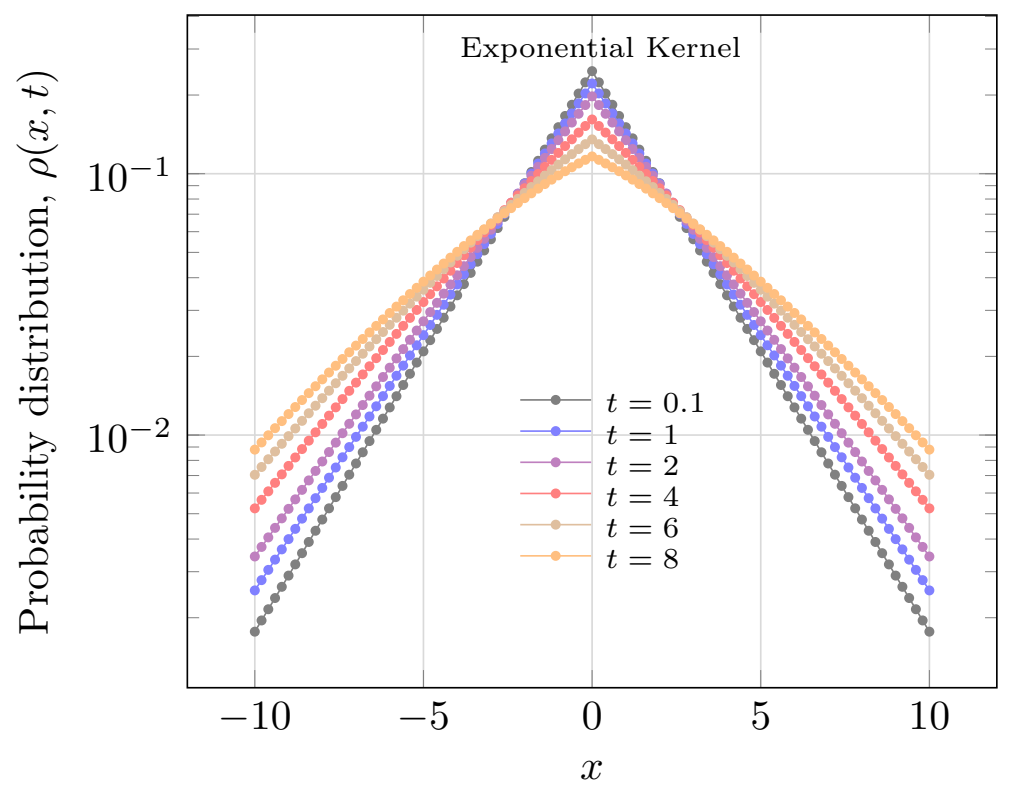

Figure 2. This curves illustrate the changes of distribution $\rho(x, t)$ caused by the exponential kernel (Equation (3) in Equation (1)), considering $\varphi(x)=\delta(x), \mathcal{D} f^{-1}=1$ and $\tau=4$ to different times $\{0.1,1,2,4,6,8\}$ (in context of Caputo-Fabrizio operator $\alpha=0.2$ and $\mathcal{D} f^{-1}=1$ ).

\subsection{Mittag-Leffler Memory-Kernel and Non-Gaussian Solutions}

The Mittag-Leffler Kernel in Laplace space is represented by kernel

$$
\mathcal{K}_{L}(s)=\frac{b s^{\alpha-1}}{\tau s^{\alpha}+1}, \quad \text { with } \quad \alpha \in(0,1)
$$

in which $\mathcal{L}\left\{E_{\alpha}\left(-\lambda t^{\alpha}\right)\right\}=\frac{s^{\alpha-1}}{\lambda+s^{\alpha}}$. The Atangana-Baleanu kernel is a special case $\left(b=\frac{b_{\alpha}}{\alpha}\right.$ and $\left.\tau=\frac{1-\alpha}{\alpha}\right)$ of the solution which is obtained in this subsection.

For the general case presented in the kernel in Equation (24), the Green function is space $(x, s)$ can be represented by Equation (20), to the next $Y(x, s)$ form

$$
Y(x, s)=\frac{1}{s} \exp \left[-\frac{|x|}{\sqrt{\tau \mathcal{D}_{\alpha} b^{-1}}} \sqrt{\frac{s^{\alpha}}{s^{\alpha}+\frac{1}{\tau}}}\right],
$$

solving the integration of branch cut associated with $s^{\alpha}$, the inverse obtained is

$$
\begin{aligned}
\mathrm{Y}(x, t) & =1-\frac{1}{\pi} \int_{0}^{\infty} e^{-t \eta} \exp \left\{-|x| \sqrt{\frac{\eta^{\alpha} b}{\tau \mathcal{D}_{\alpha} \gamma_{1}}} \cos \left[\frac{\alpha \pi-\gamma_{2}}{2}\right]\right\} \\
& \times \sin \left\{|x| \sqrt{\frac{b \eta^{\alpha}}{\mathcal{D}_{\alpha} \gamma_{1}}} \cos \left[\frac{\alpha \pi-\gamma_{2}}{2}\right]\right\} \frac{d \eta}{\eta}
\end{aligned}
$$

in that

$$
\gamma_{1}=\sqrt{\eta^{2 \alpha}+\left(\frac{1}{\tau}\right)^{2}+2\left(\frac{1}{\tau}\right) \eta^{\alpha} \cos [\alpha \pi]}
$$

and

$$
\gamma_{2}=\arctan \left[\frac{\eta^{\alpha} \sin [\alpha \pi]}{\eta^{\alpha} \cos [\alpha \pi]+\frac{1}{\tau}}\right]
$$


Using Equations (20) and (26), the $\mathcal{G}$-function to ML-memory is written as

$$
\begin{aligned}
\mathcal{G}_{2}(x, t) & =\frac{1}{2 \pi} \int_{0}^{\infty} e^{-t \eta} \exp \left\{-|x| \sqrt{\frac{b \eta^{\alpha}}{\tau \mathcal{D}_{\alpha} \gamma_{1}}} \cos \left[\frac{\alpha \pi-\gamma_{2}}{2}\right]\right\} \sqrt{\frac{b \eta^{\alpha}}{\tau \mathcal{D}_{\alpha} \gamma_{1}}} \\
& \times\left(\cos \left[\frac{\alpha \pi-\gamma_{2}}{2}\right] \sin \left\{|x| \sqrt{\frac{b \eta^{\alpha}}{\tau \mathcal{D}_{\alpha} \gamma_{1}}} \sin \left[\frac{\alpha \pi-\gamma_{2}}{2}\right]\right\}\right. \\
& \left.+\sin \left[\frac{\alpha \pi-\gamma_{2}}{2}\right] \cos \left\{|x| \sqrt{\frac{b \eta^{\alpha}}{\tau \mathcal{D}_{\alpha} \gamma_{1}}} \sin \left[\frac{\alpha \pi-\gamma_{2}}{2}\right]\right\}\right) \frac{d \eta}{\eta}
\end{aligned}
$$

which is the $\mathcal{G}$ function in $(x, t)$ space. If $\tau \rightarrow 0$ and $\alpha \rightarrow 1$, the Green function is rewritten as

$$
\mathcal{G}_{2}(x, t)=\int_{0}^{\infty} \frac{e^{-t \eta}}{\sqrt{4 \pi^{2} \mathcal{D} b^{-1} \eta}} \cos \left[\frac{x \sqrt{b \eta}}{\sqrt{\mathcal{D}}}\right] d \eta,
$$

That implies a Gaussian form to $\mathcal{G}$-function of diffusion equation. Thereby, $\rho(x, t)$ correspondent to Einstein solution to Brownian motion [1]. Figures 3 and 4 show the non-Gaussian distribution caused by different values to parameter $\alpha$ and $\tau$ in Mittag-Leffler kernel.



Figure 3. This curves illustrate the changes of distribution $\rho(x, t)$ caused by the Mittag-Leffler kernel (Equation (4) in Equation (1)), and the usual (Brownian motion) case $\mathcal{K}(t)=\delta(t)$ (or $\alpha=1$ and $\tau \rightarrow 0$ in Equation (4)), considering $\varphi(x)=\delta(x), \mathcal{D} b^{-1}=1, t=1, \tau=10^{-2}$ and different values to index $\alpha$.

The non-Gaussian shapes obtained as consequence of non-singular operator in diffusion equations can imply a rich class of non-usual diffusive systems. In next section, this hypothesis is verified. 


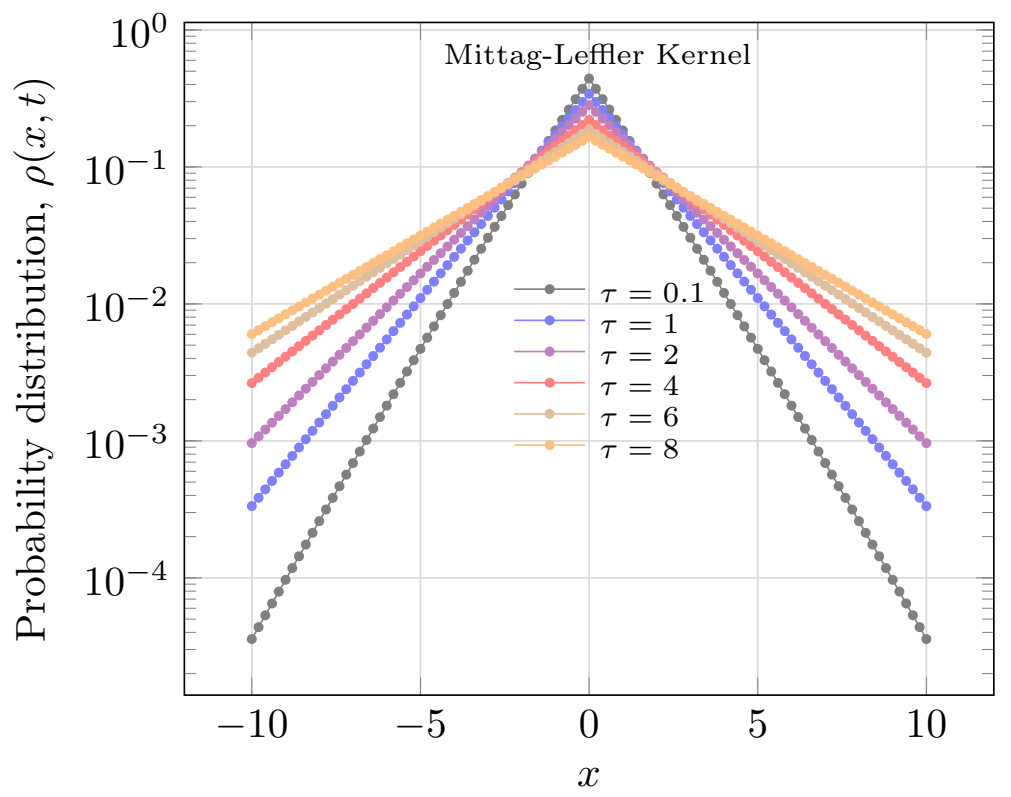

Figure 4. This curves illustrate the changes of distribution $\rho(x, t)$ caused by the Mittag-Leffler kernel (Equation (4) in Equation (1)), and the usual (Brownian motion) case $\mathcal{K}(t)=\delta(t)$ (or $\alpha=1$ and $\tau \rightarrow 0$ in Equation (4)). Considering , $\varphi(x)=\delta(x), \mathcal{D} b^{-1}=1, t=1, \alpha=0.25$ and different values of parameter $\tau$.

\subsection{Diffusive Aspects of Non-Singular Diffusion Equations}

Having established the CTRW formalism and distribution behaviours to the general situation presented here, it is relevant to obtain the temporal evolution to MSD; for symmetric distribution, we have $\left\langle(x-\langle x\rangle)^{2}\right\rangle=\left\langle x^{2}\right\rangle$. In CTRW formalism, the second moment is introduced as

$$
\left\langle x^{2}\right\rangle(t)=-\mathcal{L}^{-1}\left[\left.\frac{\partial^{2}}{\partial k^{2}} \rho(k, s)\right|_{k=0}\right] .
$$

by use of Equation (8) to localized initial condition $\varphi(k)=1$ (i.e., $\varphi(x)=\delta(x)$ ), the general form to second moment can be written as

$$
\left\langle x^{2}\right\rangle(t)=\mathcal{L}^{-1}\left[\frac{2 \mathcal{D}_{\alpha}}{s^{2} \mathcal{K}_{L}(s)}\right] .
$$

To constant kernel the second moment assume the linear time behaviour. To exponential kernel in the context of Caputo-Fabrizio diffusion, i.e., Equations (18) and (19), the inversion of Laplace transform

$$
\left\langle x^{2}\right\rangle=2 \mathcal{D}_{\alpha} f_{\alpha}^{-1}(1-\alpha+\alpha t),
$$

to the asymptotic limit of long times, this MSD assumes the linear temporal behaviour, therefore, the usual diffusion. For $\alpha=1$, we recover the standard diffusion relation $\left(\left\langle x^{2}\right\rangle=2 \mathcal{D} t\right)$. In Ref. [37], the authors investigated the fractional CF operator on Riemann-Liouville sense applied on Laplacian term of the diffusion equation, the result for distribution and MSD are very different. Thereby, the side in which the non-singular operator is applied in diffusion equation implies in different influences on diffusive properties.

To Mittag-Leffler kernel, i.e., Equation (4), the MSD implies

$$
\left\langle x^{2}\right\rangle=2 \mathcal{D}_{\alpha}\left(\tau+\frac{t^{\alpha}}{\Gamma[1+\alpha]}\right) .
$$


In the Atangana-Baleanu diffusion context, it is reduced to

$$
\left\langle x^{2}\right\rangle=2 \mathcal{D}_{\alpha} b_{\alpha}^{-1}\left(1-\alpha+\alpha \frac{t^{\alpha}}{\Gamma[1+\alpha]}\right),
$$

in which in short time the MSD of Equation (34) converges to same behaviour of MSD of Caputo-Fabrizio operator, i.e., $\left\langle x^{2}\right\rangle \propto 2 \mathcal{D} f_{\alpha}^{-1}(1-\alpha)$. In long times, Equations (34) and (35) imply an anomalous behaviour, i.e., $\left\langle x^{2}\right\rangle \sim t^{\alpha}$. This long time behaviour occurs to Caputo and Riemann-Liouville operator [38,43]. For the appropriate limit of parameters $\tau$ and $\tau$ in Equation (34), to $\tau \rightarrow 0$, we obtain the standard fractional diffusion. The diffusive behaviour to $\mathrm{CF}$ and $\mathrm{AB}$ diffusion obtained in Equations (33) and (35) recover the usual diffusive process to $\alpha \rightarrow 1$.

\subsection{Random Walk Process with Stochastic Resetting and Memory}

In this subsection, I extend the results previously obtained in another scenario. The purpose is to show the applicability of non-Gaussian forms found previously.

Diffusion with resetting is an efficient mathematical model for describing search systems [53]. This model has been the focus of many studies [54-56]. The propose of the random walk process with resetting can be resumed by expression

$$
\frac{\partial \rho}{\partial t}=\mathcal{D} \frac{\partial^{2} \rho}{\partial x^{2}}-r \rho(x, t)+r \delta\left(x-x_{0}\right), \quad \rho(x, 0)=\delta\left(x-x_{0}\right),
$$

where $r$ represents the rate of walker which are removed on system and restart in the initial condition. This idea can maximize the search for targets in a specific region. Some extensions of this model has been investigate on Levy flight context [57], coagulation-diffusion process [58] and others. The purpose is to generalise Equation (36) in the context of memory kernel, as follows

$$
\int_{0}^{t} d t^{\prime} \gamma\left(t-t^{\prime}\right) \frac{\partial \rho}{\partial t^{\prime}}=\mathcal{D} \frac{\partial^{2} \rho}{\partial x^{2}}-r \int_{0}^{t} d t^{\prime} \gamma\left(t^{\prime}\right)\left(\rho\left(x, t-t^{\prime}\right)-\delta\left(x-x_{0}\right)\right),
$$

with truncated memory kernel

$$
\gamma(t)=e^{-r t} \mathcal{K}(t),
$$

where $r=0$. Equation (37) recovers Equation (1). If $\mathcal{K}(t)=\delta(t)$, Equation (37) implies Equation (36). To solve the proposed model, I use the technique present in previous section, which implies on solution in Fourier-Laplace space, as

$$
\rho(k, s)=\frac{1}{s} \frac{\mathcal{K}_{L}(s+r)(s+r) e^{-i k x_{0}}}{(s+r) \mathcal{K}_{L}(s+r)+k^{2} \mathcal{D}}
$$

where $\mathcal{L}\left\{\mathcal{K}(t) e^{-r t}\right\}=\mathcal{K}_{L}(s+r)$, the systems have normalized number of walkers, i.e., $\int_{-\infty}^{+\infty} \rho(x, t) d x=1$. How $\lim _{t \rightarrow \infty} \rho(k, t)=\mathcal{L}^{-1}\left\{\lim _{s \rightarrow 0} \rho(k, s)\right\}$, to kernels considered in this work, i.e., Equations (18)-(24), we have

$$
\rho(k, t)=\frac{\mathcal{K}_{L}(r) r e^{-i k x_{0}}}{r \mathcal{K}_{L}(r)+k^{2} \mathcal{D}}
$$

performing the inverse Fourier transform, we obtain the general stationary solution

$$
\rho_{s}(x)=\beta \frac{e^{-\beta\left|x-x_{0}\right|}}{2}, \quad \beta=\sqrt{\frac{r \mathcal{K}_{L}(r)}{\mathcal{D}}},
$$


in which $\mathcal{K}_{L}$ are functions presented in Sections 3.1 and 3.2. To $\mathcal{K}_{L}(s)=1$, i.e., $\mathcal{K}(t)=\delta(t)$, we recover the usual case reported in Ref. [53].

The inverse transformations of Fourier and Laplace of Equation (39) to exponential kernel (Equation (3)) implies

$$
\begin{aligned}
\rho(x, t) & =\mathcal{L}^{-1}\left[\left(1+\frac{r}{s}\right) e^{-i k x_{0}} \mathcal{G}_{1}(k, s+r)\right] \\
& =e^{-r t} \mathcal{G}_{1}\left(x-x_{0}, t\right)+r \int_{0}^{t} d t^{\prime} e^{-r t^{\prime}} \mathcal{G}_{1}\left(x-x_{0}, t^{\prime}\right),
\end{aligned}
$$

in which $\mathcal{G}_{1}$ was obtained in Equation (23). To diffusion resetting problem of random walks in the context of exponential kernel, the MSD can be written as follows

$$
\left\langle x^{2}\right\rangle(t)=2 \frac{\mathcal{D}}{f}\left(\tau-\frac{1}{r}\right) e^{-r t}+\frac{2 \mathcal{D}}{r f},
$$

with $x_{0}=0$. To $r \rightarrow 0$, we obtain $\left\langle x^{2}\right\rangle(t)=2 \mathcal{D} f^{-1}(\tau+t)$. It is clear that, for small time, we recover the initial behaviour to walkers present in Section 3.3. To long times in Equation (43), we obtain a constant MSD behaviour, which is justified by a stationary distribution (Equation (41)) that represents a delicate equilibrium between added and removed walkers.

On the other hand, to Mittag-Leffler kernel, i.e., Equation (4), we have the same mathematical structure of Equation (42), as follows

$$
\rho(x, t)=e^{-r t} \mathcal{G}_{2}\left(x-x_{0}, t\right)+r \int_{0}^{t} d t^{\prime} e^{-r t^{\prime}} \mathcal{G}_{2}\left(x-x_{0}, t^{\prime}\right),
$$

in which $\mathcal{G}_{2}$ was obtained in Equation (30). Performing the calculation of MSD to random walkers under effect of Mittag-Leffler kernel, we obtain

$$
\left\langle x^{2}\right\rangle(t)=2 \frac{\mathcal{D}}{b} \tau+2 \mathcal{D} \frac{(\Gamma(\alpha)-\Gamma(\alpha, r t))}{b r^{\alpha} \Gamma(\alpha)},
$$

with $x_{0}=0$ and $\Gamma(\alpha, z)=\int_{z}^{\infty} d t t^{\alpha-1} e^{-t}$. Figure 5 illustrates the temporal evolution of Equation (45) for a set of different $\alpha$ parameters. The figure reveals a rich class of sub-diffusive behaviours to process. Equation (45) implies in an extensive class of behaviour associated with rate $r$ of added and removed walkers.

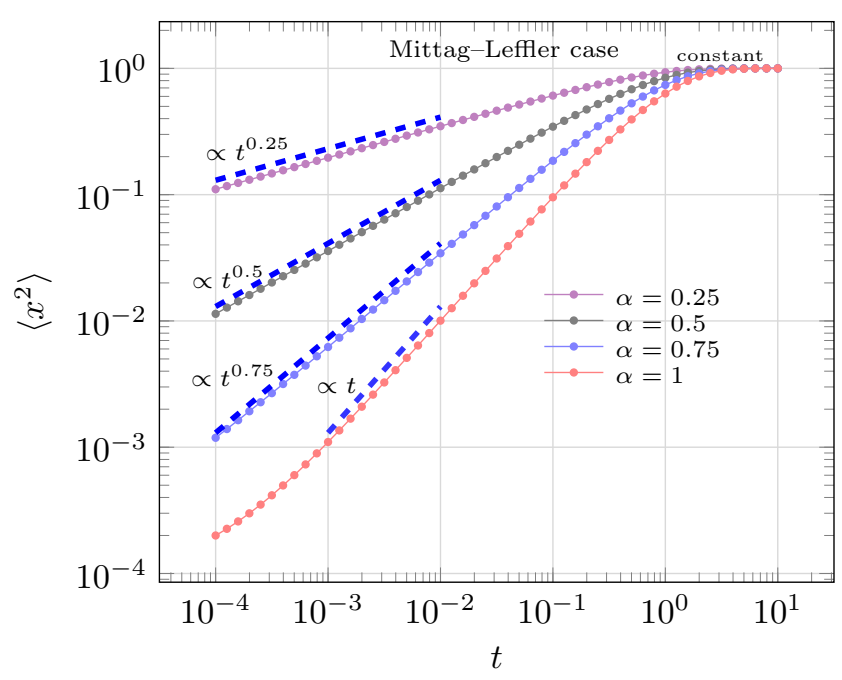

Figure 5. These curves illustrate the changes of MSD caused by a set of fractional $\alpha$-index in Mittag-Leffler kernel, considering $\tau=10^{-4}, \mathcal{D} b^{-1}=0.5$ and $r=1$. 
The problem presented in this subsection makes clear that the non-Gaussian solutions found in Sections 3.1 and 3.2 have an important degree of applicability. The next step is to apply the techniques presented in this work to investigate the effectiveness of search strategies for single and multiple targets, as investigated in Ref. [59]; thereby, the solutions found in this work can be very interesting for the investigation of other problems in mathematical models.

\section{Conclusions}

The fractional diffusion equations exhibit a very complex behaviour and its properties and anomalies are strongly influenced by memory effects or generalised waiting time distribution in Montroll-Weiss equation. In this sense, it is possible to write different forms of the diffusion equation. This work approaches the mathematical model expressed by Equation (1) and seeks to present this proposal generates compact solutions for walkers under memory effect. In Section 3, I present the exact solutions of the generalised diffusion equation and the time dependence of the mean square displacement to different memory kernels. The results reveal that memory kernels lead to non-Gaussian distributions (Sections 3.1 and 3.2) and different diffusive regimes to MSD depending on the timescale (Section 3.3). For the Mittag-Leffler kernel, a crossover between two diffusive regimes occurs: constant for small times and sub-diffusive for long times, a feature observed in several empirical systems. In addition, I show the role of each of these kernels in the new forms of diffusion equations in the context of $\mathrm{CF}$ and $\mathrm{AB}$ fractional operators on Caputo sense. Moreover, in Section 3.4, I generalise the random walk process with stochastic resetting. The problem reveals a series of new consequences in relation to the usual case, including sub-diffusive process regimes $\left(\left\langle x^{2}\right\rangle \sim t^{\alpha}\right.$ with $\left.\alpha<1\right)$ due to Mittag-Leffler kernel, and shows the applicability of the solutions obtained in the previous sections in another scenario.

The results and methods used in this work to approach non-singular kernels in diffusion equation suggest a simple way for incorporating the memory effects on description of dynamic behaviour with non-usual aspects. Thereby, this work reveals simple forms to solutions for generalised diffusion equation with memory kernels (exponential and Mittag-Leffler functions). Consequently, the solutions appear as a simple alternative to approach mathematical models associated with fractional operators of Caputo-Fabrizio and Atangana-Baleanu in diffusion problems. Moreover, this work presents possibilities for future investigations of diffusion in fractal systems, intermittent movement and reaction-diffusion systems, under influence of non-singular memory kernels.

Funding: This research received no external funding.

Acknowledgments: I thank the Brazilian National Council for Scientific and Technological Development (CNPq). I gratefully thank the referees for their comments and recommendations.

Conflicts of Interest: The author declares no conflict of interest.

\section{References}

1. Einstein, A. Über die von der molekularkinetischen Theorie der Wärme geforderte Bewegung von in ruhenden Flüssigkeiten suspendierten Teilchen. Ann. Phys. 1905, 322, 549-560. [CrossRef]

2. Von Smoluchowski, M. Zur kinetischen theorie der brownschen molekularbewegung und der suspensionen. Ann. Phys. 1906, 326, 756-780. [CrossRef]

3. Langevin, P. Sur la théorie du mouvement brownien. CR Acad. Sci. Paris 1908, 146, 530.

4. Perrin, J. L'agitation moléculaire et le mouvement brownien. Comptes Rendus Hebdomadaires des Séances de L'académie des Sciences 1908, 146, 967-970.

5. Perrin, J. Mouvement brownien et réalité moléculaire. Annales de Chimie et de Physique 1909, 18, 5-104.

6. Alves, L.G.; Winter, P.B.; Ferreira, L.N.; Brielmann, R.M.; Morimoto, R.I.; Amaral, L.A. Long-range correlations and fractal dynamics in C. elegans: Changes with aging and stress. Phys. Rev. E 2017, 96, 022417. [CrossRef] [PubMed] 
7. $\quad$ Alves, L.G.; Scariot, D.B.; Guimarães, R.R.; Nakamura, C.V.; Mendes, R.S.; Ribeiro, H.V. Transient superdiffusion and long-range correlations in the motility patterns of trypanosomatid flagellate protozoa. PLoS ONE 2016, 11, e0152092. [CrossRef] [PubMed]

8. Reverey, J.F.; Jeon, J.H.; Bao, H.; Leippe, M.; Metzler, R.; Selhuber-Unkel, C. Superdiffusion dominates intracellular particle motion in the supercrowded cytoplasm of pathogenic Acanthamoeba castellanii. Sci. Rep. 2015, 5, 11690. [CrossRef] [PubMed]

9. Ribeiro, H.V.; Tateishi, A.A.; Alves, L.G.; Zola, R.S.; Lenzi, E.K. Investigating the interplay between mechanisms of anomalous diffusion via fractional Brownian walks on a comb-like structure. New J. Phys. 2014, 16, 093050. [CrossRef]

10. Metzler, R.; Jeon, J.H.; Cherstvy, A.G.; Barkai, E. Anomalous diffusion models and their properties: Non-stationarity, non-ergodicity, and ageing at the centenary of single particle tracking. Phys. Chem. Chem. Phys. 2014, 16, 24128-24164. [CrossRef] [PubMed]

11. Vahabi, M.; Schulz, J.H.; Shokri, B.; Metzler, R. Area coverage of radial Lévy flights with periodic boundary conditions. Phys. Rev. E 2013, 87, 042136. [CrossRef] [PubMed]

12. Sibatov, R.; Uchaikin, V. Dispersive transport of charge carriers in disordered nanostructured materials. J. Comput. Phys. 2015, 293, 409-426. [CrossRef]

13. Shikano, Y.; Wada, T.; Horikawa, J. Discrete-time quantum walk with feed-forward quantum coin. Sci. Rep. 2014, 4, 4427. [CrossRef] [PubMed]

14. Dos Santos Mendes, R.; Lenzi, E.K.; Malacarne, L.C.; Picoli, S.; Jauregui, M. Random Walks Associated with Nonlinear Fokker-Planck Equations. Entropy 2017, 19, 155. [CrossRef]

15. Plastino, A.; Curado, E.; Nobre, F.; Tsallis, C. From the nonlinear Fokker-Planck equation to the Vlasov description and back: Confined interacting particles with drag. Phys. Rev. E 2018, 97, 022120. [CrossRef] [PubMed]

16. Mendes, G.; Ribeiro, M.; Mendes, R.; Lenzi, E.; Nobre, F. Nonlinear Kramers equation associated with nonextensive statistical mechanics. Phys. Rev. E 2015, 91, 052106. [CrossRef] [PubMed]

17. Chen, W.B.; Wang, J.; Qiu, W.Y.; Ren, F.Y. Solutions for a time-fractional diffusion equation with absorption: influence of different diffusion coefficients and external forces. J. Phys. A 2008, 41, 045003. [CrossRef]

18. Podlubny, I. Fractional Differential Equations: An Introduction to Fractional Derivatives, Fractional Differential Equations, to Methods of Their Solution and Some of Their Applications; Elsevier: New York, NY, USA, 1998; Volume 198.

19. Yang, X.J.; Machado, J.T.; Baleanu, D. Anomalous diffusion models with general fractional derivatives within the kernels of the extended Mittag-Leffler type functions. Rom. Rep. Phys. 2017, 69, 115.

20. Sabatier, J.; Agrawal, O.P.; Machado, J.T. Advances in Fractional Calculus; Springer: Berlin, Germany, 2007; Volume 4 .

21. Ha, S.Y.; Jung, J. Remarks on the slow relaxation for the fractional Kuramoto model for synchronization. J. Math. Phys. 2018, 59, 032702. [CrossRef]

22. Batool, F.; Akram, G. A novel approach for solitary wave solutions of the generalized fractional ZakharovKuznetsov equation. Indian J. Phys. 2018, 92, 111-119. [CrossRef]

23. Lenzi, E.K.; dos Santos, M.A.F.; Lenzi, M.K.; Neto, R.M. Solutions for a mass transfer process governed by fractional diffusion equations with reaction terms. Commun. Nonlinear Sci. Numer. Simul. 2017, 48, 307-317. [CrossRef]

24. Dos Santos, M.A.F.; Lenzi, M.K.; Lenzi, E.K. Anomalous Diffusion with an Irreversible Linear Reaction and Sorption-Desorption Process. Adv. Math. Phys. 2017, 2017. [CrossRef]

25. Lenzi, E.K.; Ribeiro, H.V.; dos Santos, M.A.F.; Rossato, R.; Mendes, R.S. Time dependent solutions for a fractional Schrödinger equation with delta potentials. J. Math. Phys. 2013, 54, 082107. [CrossRef]

26. Carpinteri, A.; Mainardi, F. Fractals and Fractional Calculus in Continuum Mechanics; Springer: Berlin, Germany, 2014; Volume 378.

27. Liemert, A.; Sandev, T.; Kantz, H. Generalized Langevin equation with tempered memory kernel. Physica A 2017, 466, 356-369. [CrossRef]

28. Liemert, A.; Kienle, A. Fractional radiative transport in the diffusion approximation. J. Math. Chem. 2018, 56, 317-335. [CrossRef]

29. Liang, Y.; Chen, W. Continuous time random walk model with asymptotical probability density of waiting times via inverse Mittag-Leffler function. Commun. Nonlinear Sci. Numer. Simul. 2018, 57, 439-448. [CrossRef] 
30. Le Vot, F.; Abad, E.; Yuste, S. Continuous-time random-walk model for anomalous diffusion in expanding media. Phys. Rev. E 2017, 96, 032117. [CrossRef] [PubMed]

31. Atangana, A.; Baleanu, D. New fractional derivatives with nonlocal and non-singular kernel: Theory and application to heat transfer model. Therm. Sci. 2016, 18. [CrossRef]

32. Caputo, M.; Fabrizio, M. A new definition of fractional derivative without singular kernel. Progr. Fract. Differ. Appl. 2015, 1, 1-13.

33. Baleanu, D.; Jajarmi, A.; Hajipour, M. On the nonlinear dynamical systems within the generalized fractional derivatives with Mittag-Leffler kernel. Nonlinear Dyn. 2018, 1-18. [CrossRef]

34. Hristov, J. Steady-state heat conduction in a medium with spatial non-singular fading memory: Derivation of Caputo-Fabrizio space-fractional derivative with Jeffrey's kernel and analytical solutions. Therm. Sci. 2016, 00, 115. [CrossRef]

35. Hristov, J. Derivatives with non-singular kernels from the Caputo-Fabrizio definition and beyond: Appraising analysis with emphasis on diffusion models. Front. Fract. Calc. 2017, 1, 270-342.

36. Sandev, T.; Sokolov, I.M.; Metzler, R.; Chechkin, A. Beyond monofractional kinetics. Chaos Solitons Fractals 2017, 102, 210-217. [CrossRef]

37. Tateishi, A.A.; Ribeiro, H.V.; Lenzi, E.K. The role of fractional time-derivative operators on anomalous diffusion. Front. Phys. 2017, 5, 52. [CrossRef]

38. Sandev, T.; Chechkin, A.; Kantz, H.; Metzler, R. Diffusion and Fokker-Planck-Smoluchowski equations with generalized memory kernel. Fract. Calc. Appl. Anal. 2015, 18, 1006-1038. [CrossRef]

39. Montroll, E.W.; Weiss, G.H. Random walks on lattices. II. J. Math. Phys. 1965, 6, 167-181. [CrossRef]

40. Scher, H.; Montroll, E.W. Anomalous transit-time dispersion in amorphous solids. Phys. Rev. B 1975, $12,2455$. [CrossRef]

41. Shlesinger, M.F. Origins and applications of the Montroll-Weiss continuous time random walk. Eur. Phys. J. B 2017, 90, 93. [CrossRef]

42. Bouchaud, J.P.; Georges, A. Anomalous diffusion in disordered media: Statistical mechanisms, models and physical applications. Phys. Rep. 1990, 195, 127-293. [CrossRef]

43. Metzler, R.; Klafter, J. The random walk's guide to anomalous diffusion: A fractional dynamics approach. Phys. Rep. 2000, 339, 1-77. [CrossRef]

44. Aghion, E.; Kessler, D.A.; Barkai, E. Asymptotic densities from the modified Montroll-Weiss equation for coupled CTRWs. Eur. Phys. J. B 2018, 91, 17. [CrossRef]

45. Yadav, A.; Fedotov, S.; Méndez, V.; Horsthemke, W. Propagating fronts in reaction-transport systems with memory. Phys. Lett. A 2007, 371, 374-378. [CrossRef]

46. Ben-Zvi, R.; Nissan, A.; Scher, H.; Berkowitz, B. A continuous time random walk (CTRW) integro-differential equation with chemical interaction. Eur. Phys. J. B 2018, 91, 15. [CrossRef]

47. Furnival, T.; Leary, R.K.; Tyo, E.C.; Vajda, S.; Ramasse, Q.M.; Thomas, J.M.; Bristowe, P.D.; Midgley, P.A. Anomalous diffusion of single metal atoms on a graphene oxide support. Chem. Phys. Lett. 2017, 683, 370-374. [CrossRef]

48. Montroll, E.W.; Scher, H. Random walks on lattices. IV. Continuous-time walks and influence of absorbing boundaries. J. Stat. Phys. 1973, 9, 101-135. [CrossRef]

49. Sandev, T.; Metzler, R.; Chechkin, A. From continuous time random walks to the generalized diffusion equation. Fract. Calc. Appl. Anal. 2018, 21, 10-28. [CrossRef]

50. Mainardi, F.; Pagnini, G.; Saxena, R. Fox H functions in fractional diffusion. J. Comput. Appl. Math. 2005, 178, 321-331. [CrossRef]

51. Barenblatt, G.; Zheltov, I.P.; Kochina, I. Basic concepts in the theory of seepage of homogeneous liquids in fissured rocks [strata]. J. Appl. Math. Mech. 1960, 24, 1286-1303. [CrossRef]

52. Prudnikov, A.P.; Marichev, O.I. Integrals and Series. Vol. 4' Laplace Transforms; Gordon and Breach Science Publishers: New York, NY, USA; London, UK; Tokyo, Japan, 1992; Volume 18.

53. Evans, M.R.; Majumdar, S.N. Diffusion with stochastic resetting. Phys. Rev. Lett. 2011, $106,160601$. [CrossRef] [PubMed]

54. Chatterjee, A.; Christou, C.; Schadschneider, A. Diffusion with resetting inside a circle. Phys. Rev. E 2018, 97, 062106. [CrossRef] [PubMed]

55. Pal, A.; Kundu, A.; Evans, M.R. Diffusion under time-dependent resetting. J. Phys. A Math. Theor. 2016, 49, 225001. [CrossRef] 
56. Falcao, R.; Evans, M.R. Interacting Brownian motion with resetting. J. Stat. Mech. Theory Exp. 2017, $2017,023204$. [CrossRef]

57. Kuśmierz, Ł.; Gudowska-Nowak, E. Optimal first-arrival times in Lévy flights with resetting. Phys. Rev. E 2015, 92, 052127.

58. Durang, X.; Henkel, M.; Park, H. The statistical mechanics of the coagulation-diffusion process with a stochastic reset. J. Phys. A Math. Theor. 2014, 47, 045002. [CrossRef]

59. Palyulin, V.V.; Mantsevich, V.N.; Klages, R.; Metzler, R.; Chechkin, A.V. Comparison of pure and combined search strategies for single and multiple targets. Eur. Phys. J. B 2017, 90, 170. [CrossRef]

(C) 2018 by the authors. Licensee MDPI, Basel, Switzerland. This article is an open access article distributed under the terms and conditions of the Creative Commons Attribution (CC BY) license (http://creativecommons.org/licenses/by/4.0/). 